\title{
Risk factors for requirement of filtration surgery after vitrectomy in patients with proliferative diabetic retinopathy
}

This article was published in the following Dove Press journal:

Clinical Ophthalmology

\section{Masashi Sakamoto \\ Ryuya Hashimoto \\ Izumi Yoshida \\ Takatoshi Maeno}

Department of Ophthalmology, Sakura Medical Center of Toho University,

Shimoshizu, Sakura, Japan
Correspondence: Masashi Sakamoto Department of Ophthalmology, Toho University Sakura Medical Center, 564-I Shimoshizu, Sakura-shi, Chiba Prefecture 285-874I, Japan

$\mathrm{Tel}+8 \mathrm{I} 4346288 \mathrm{II}$

Fax $+8 \mid 434628820$

Email masashi.sakamoto@med. toho-u.ac.jp
Purpose: We retrospectively reviewed patients with postoperative neovascular glaucoma (NVG) after vitrectomy for proliferative diabetic retinopathy to investigate how variables assessed before, during, and after vitrectomy are associated with the requirement for filtration surgery.

Patients and methods: The subjects in this retrospective, observational, comparative study were 55 consecutive patients (61 eyes) who underwent vitrectomy for proliferative diabetic retinopathy at Toho University Sakura Medical Center between December 2011 and November 2016, were followed up for at least 6 months after surgery, and developed NVG within 2 years after surgery. They comprised 44 men and 11 women of mean age 52.4 \pm 9.1 years, who were followed up for a mean $7.1 \pm 6.1$ months. We collected data on the following 16 variables: sex, age, history of panretinal photocoagulation completed within 3 months before vitrectomy, presence/absence of a lens, obvious iris/angle neovascularization, tractional retinal detachment, diabetic macular edema, vitreous hemorrhage, visual acuity and intraocular pressure before vitrectomy and at the onset of NVG, glycated hemoglobin, fasting blood glucose, estimated glomerular filtration rate, and use of intraoperative gas tamponade.

Results: Logistic regression analysis with the backward elimination method identified preoperative fasting hyperglycemia $(P=0.08)$, high intraocular pressure at the onset of NVG $(P=0.04)$, and use of gas tamponade during vitrectomy $(P=0.008)$ to be significant risk factors for requirement of filtration surgery.

Conclusion: Preoperative fasting hyperglycemia, high intraocular pressure at the onset of NVG, and use of gas tamponade during vitrectomy predispose patients to require filtration surgery in the event of postoperative NVG.

Keywords: neovascular glaucoma, proliferative diabetic retinopathy, vitrectomy, gas tamponade

\section{Introduction}

Vitrectomy for proliferative diabetic retinopathy (PDR) has been reported to be associated with postoperative neovascular glaucoma (NVG) in $4 \%-12 \%$ of cases. ${ }^{1-5}$ Although an improved treatment outcome has been reported, NVG remains a potential cause of severe visual impairment. Previous studies have identified several risk factors for NVG after vitrectomy, including obvious preoperative neovascularization of the iris/angle, male sex, and postoperative retinal detachment. ${ }^{3,6,7}$ The reported treatments for NVG include intravitreal injection of an anti-vascular endothelial growth factor (anti-VEGF) agent, ${ }^{8-10}$ additional retinal photocoagulation, and trabeculectomy or tube shunt surgery for closed-angle NVG or other conditions unresponsive to intraocular pressure (IOP) reduction therapy. ${ }^{11,12}$ 
However, to the best of our knowledge, risk factors for the requirement of filtration surgery after vitrectomy in patients with PDR have not yet been examined. The aim of this study was to investigate how variables assessed before, during, and after vitrectomy are associated with the requirement of filtration surgery in patients who develop NVG after PDR surgery.

\section{Patients and methods}

This retrospective, observational, comparative study included the medical records of 55 consecutive patients (61 eyes) who underwent vitrectomy for PDR at Toho University Sakura Medical Center between December 2011 and November 2016, were followed for at least 6 months postoperatively, and developed NVG within 2 years after surgery (44 men, 11 women, mean age 52.4 9.1 years, mean follow-up 7.1 \pm 6.1 months). All eyes had PDR; we performed pars plana vitrectomy in eyes with PDR and diabetic macular edema, but not in eyes that had diabetic macular edema alone. The follow-up period was defined as the duration from the day of vitrectomy to the first documentation of NVG. When IOP was first noted to be raised after pars plana vitrectomy, we searched for neovascularization of the iris with the slit lamp. If no neovascularization was present, gonioscopy was performed. The diagnosis of NVG was based on the presence of iris/angle neovascularization and an IOP $\geq 22 \mathrm{mmHg}$. Patients were divided into 2 groups as follows: a filtration surgery $(+)$ group that included patients in whom filtration surgery was indicated who either underwent filtration surgery or refused to undergo surgery and lost their vision and a filtration surgery (-) group that included patients who had their IOP controlled at or below $21 \mathrm{mmHg}$ by intravitreal injection of anti-VEGF agents, additional retinal photocoagulation, or instillation of glaucoma eye drops and did not require filtration surgery. We did not perform cyclodestruction in eyes that required filtration surgery in this study or in the filtration $(-)$ group. We retrospectively reviewed the patients' records for the following variables: sex, age, history of panretinal photocoagulation (PRP) completed within 3 months before vitreous surgery, presence/absence of a lens, obvious iris/angle neovascularization, tractional retinal detachment (TRD), diabetic macular edema, vitreous hemorrhage, preoperative $\log$ MAR visual acuity, IOP, glycated hemoglobin $\left(\mathrm{HbA}_{1 \mathrm{c}}\right)$, fasting blood glucose, and estimated glomerular filtration rate (eGFR) as preoperative factors; use of gas tamponade as an intraoperative factor; and IOP and $\log$ MAR visual acuity at the onset of NVG as postoperative factors.

The study protocol was approved by the Institutional Review board service at Toho University Sakura Medical
Center (approval number No S17005). The procedures used conformed to the tenets of the Declaration of Helsinki. The design of the study was explained to patients using the Toho University Sakura Medical Center website in accordance with the guidelines for clinical research set out by the Japanese Ministry of Health, Labour, and Welfare. All patients provided written informed consent prior to initiation of any treatment. Any and all private patient information were excluded from the database, and use of these anonymous data was approved by the Institutional Review Board services without the need to seek another consent for use of data for research.

\section{Surgical procedure}

Lens removal, if necessary, was performed by phacoemulsification or pars plana lensectomy. The former procedure was followed by intraocular lens implantation in the posterior chamber, and the latter procedure was followed by intraocular lens implantation over the anterior capsule immediately or later on another day. Vitreous surgery was performed using a 20-gauge or a 25-gauge 3-port system. Completion of vitrectomy was followed by removal of the fibrovascular proliferative membrane and cauterization of the retinal neovessels to create a posterior vitreous detachment to the furthest periphery possible. The vitreous base was then shaved via scleral compression, followed by PRP achieved using intraocular laser treatment.

\section{Statistical analyses}

The statistical analyses were performed using IBM SPSS Statistics version 24 (IBM Corporation, Armonk, NY, USA) software. The 2 groups were assessed for sex-related differences using the Mann-Whitney $U$ test. Between-group comparisons for history of PRP, presence/absence of a lens before surgery, obvious iris/angle neovascularization, TRD, diabetic macular edema, vitreous hemorrhage, and use of gas tamponade were performed using Fisher's exact test. Between-group comparisons for age, IOP, and visual acuity before vitreous surgery and at the onset of $\mathrm{NVG}, \mathrm{HbA}_{1 \mathrm{c}}$, fasting blood glucose, eGFR, and time to onset of postoperative NVG were conducted using the Mann-Whitney $U$ test. The number of preoperative intravitreal bevacizumab injections, the number of these injections after surgery, and PPV incision size were compared between groups using the chi-squared test.

The following 16 variables were subjected to logistic regression analysis: sex, age, history of PRP, presence/ absence of a lens before surgery, obvious iris/angle neovascularization before vitreous surgery, TRD, diabetic macular 
edema, vitreous hemorrhage, intraoperative tamponade, visual acuity and IOP before vitreous surgery and at NVG onset, $\mathrm{HbA}_{1 \mathrm{c}}$, fasting blood glucose, and eGFR. Fasting blood glucose was identified as a significant variable $(P<0.05)$. The variables were then subjected to the backward elimination method to identify the risk factors for requirement of filtration surgery. A $P$-value of $<0.05$ was considered to be statistically significant.

\section{Results}

The filtration surgery (+) and filtration surgery (-) groups comprised 40 and 21 eyes, respectively. For eyes with retinal detachment or an artificially created retinal tear, retinal tamponade was achieved using air, $20 \% \mathrm{SF}_{6}$, or $12 \% \mathrm{C}_{3} \mathrm{~F}_{8}$. Tamponade was performed in 28 patients in the filtration surgery (+) group using $20 \% \mathrm{SF}_{6}, 12 \% \mathrm{C}_{3} \mathrm{~F}_{8}$, and air in 15 , 6 , and 7 patients, respectively; in the filtration surgery (-) group, tamponade was performed in 4 patients using $20 \%$ $\mathrm{SF}_{6}$ and in 1 patient using $12 \% \mathrm{C}_{3} \mathrm{~F}_{8}$.

Univariate analysis identified use of gas tamponade, preoperative fasting hyperglycemia, poor postoperative $\operatorname{logMAR}$ visual acuity, and high IOP at the onset of NVG to be significant predictors of requirement for filtration surgery. The mean time to NVG onset was significantly shorter in the filtration surgery $(+)$ group $(5.1 \pm 3.9$ months $)$ than in the filtration (-) group ( $11 \pm 7.6$ months; $P<0.05$, Welch's test; Table 1). Logistic regression analysis identified fasting blood glucose as a significant variable $(P<0.05$; Table 2$)$. The backward elimination method for variables with $P<0.2$ in logistic regression analysis identified preoperative fasting hyperglycemia, high IOP at the onset of NVG, and use of gas tamponade intraoperatively $(P<0.05$; Table 3$)$ as significant risk factors that predispose patients to needing filtration surgery in the event of postoperative NVG.

\section{Discussion}

In this study, we identified preoperative fasting blood glucose as a factor, postoperative IOP at the onset of NVG, and intraoperative use of gas tamponade to be significant predictors of the requirement for filtration surgery in the event of NVG after vitrectomy for PDR.

High IOP at the onset of NVG suggests organic angle obstruction and trabecular dysfunction in patients with $\mathrm{NVG}$ after PDR vitrectomy who are unresponsive to IOP reduction therapy, such as intravitreal injection of anti-VEGF agents and additional retinal photocoagulation. This condition leads to requirement of filtration surgery in these patients.

In general, gas tamponade is used when an iatrogenic retinal tear is created during PDR surgery or retinal detachment
Table I Patient demographic characteristics

\begin{tabular}{|c|c|c|c|}
\hline & $\begin{array}{l}\text { Filtration } \\
\text { surgery }(+)\end{array}$ & $\begin{array}{l}\text { Filtration } \\
\text { surgery (-) }\end{array}$ & $P$-value \\
\hline $\mathrm{n}$ & 40 & 21 & - \\
\hline Male sex, \% & 85 & 71.4 & 0.31 \\
\hline Age, years & $51.9 \pm 10.3$ & $53.3 \pm 6.2$ & 0.69 \\
\hline PRP (+), \% & 62.5 & 76.2 & 0.39 \\
\hline $\begin{array}{l}\text { Presence of lens before } \\
\text { vitrectomy, \% }\end{array}$ & 85 & 95.2 & 0.40 \\
\hline Rubeosis, \% & 10 & 14.3 & 0.68 \\
\hline TRD (+), \% & 42.5 & 23.8 & 0.17 \\
\hline ME (+), \% & 15 & 23.8 & 0.49 \\
\hline $\mathrm{VH}(+), \%$ & 52.5 & 66.7 & 0.41 \\
\hline Use of gas tamponade, $\%$ & 70 & 23.8 & $<0.01$ \\
\hline Preoperative logMAR & $1.21 \pm 0.67$ & $1.36 \pm 0.60$ & 0.37 \\
\hline Preoperative IOP, $\mathrm{mmHg}$ & $14.8 \pm 3.4$ & $15.4 \pm 3.3$ & 0.39 \\
\hline LogMAR after vitrectomy & $1.43 \pm 0.8$ & $0.90 \pm 0.8$ & 0.02 \\
\hline IOP after vitrectomy, $\mathrm{mmHg}$ & $37.3 \pm 11.6$ & $30.7 \pm 7.8$ & 0.03 \\
\hline $\mathrm{HbA}_{1 \mathrm{c}}, \%$ & $7.1 \pm 1.4$ & $7.4 \pm 1.7$ & 0.56 \\
\hline $\mathrm{FBG}, \mathrm{mg} / \mathrm{dL}$ & $148.0 \pm 39.6$ & $195.9 \pm 72.0$ & $<0.01$ \\
\hline eGFR, $\mathrm{mL} / \mathrm{min} / 1.73 \mathrm{~m}^{2}$ & $69.4 \pm 26.1$ & $82.3 \pm 29.2$ & 0.12 \\
\hline Mean time to NVG onset, months & $5.1 \pm 3.9$ & $11 \pm 7.6$ & $<0.01$ \\
\hline Preoperative IVB, \% & 10 & 15 & 0.54 \\
\hline IVB immediately after surgery, \% & 10 & 3 & 0.23 \\
\hline $\begin{array}{l}\text { Incision size of PPV ( } 25 G \\
\text { vs } 20 \mathrm{G}), \%\end{array}$ & 67 & 73 & 0.64 \\
\hline
\end{tabular}

Notes: Data are expressed as mean \pm SD. The proportion of male patients was analyzed by Fisher's exact test. Between-group comparisons for a history of PRP, presence/absence of lens before surgery, obvious iris/angle neovascularization, TRD, diabetic macular edema, vitreous hemorrhage, and use of gas tamponade were analyzed by Fisher's exact test. The values for age, IOP, and visual acuity before vitreous surgery and at NVG onset, $\mathrm{HbA}_{\mathrm{lc}}$, FBG, eGFR, and time to onset of postoperative NVG were analyzed by the Mann-Whitney $U$ test. Between-group comparisons of preoperative IVB, IVB after surgery, and PPV incision size were analyzed by the chi-squared test.

Abbreviations: eGFR, estimated glomerular filtration rate; FBG, fasting blood glucose; $\mathrm{HbA}_{\mathrm{Ic}}$, glycated hemoglobin; IOP, intraocular pressure; IVB, intravitreal bevacizumab; ME, macular edema; NVG, neovascular glaucoma; PPV, pars plana vitrectomy; PRP, panretinal photocoagulation; TRD, tractional retinal detachment; $\mathrm{VH}$, vitreous hemorrhage.

has occurred. Conflicting data have been reported with regard to the efficacy of gas tamponade, that is, a decreased incidence of early vitreous hemorrhage ${ }^{13}$ and an unchanged incidence of vitreous hemorrhage. ${ }^{14,15} \mathrm{~A}$ postoperative increase in IOP has also been reported in patients who have undergone vitrectomy for an idiopathic macular hole ${ }^{16}$ or rhegmatogenous retinal detachment, in whom postoperative inflammation may lead to increased IOP in the late postoperative phase. ${ }^{17}$ In this study, gas tamponade was identified as a significant risk factor for requirement of filtration surgery. Previous studies have reported increased VEGF levels in aqueous humor in patients with NVG. ${ }^{18-20}$ A possible mechanism is inflammation of the anterior chamber in response to gas tamponade and subsequent angle neovascularization induced by inflammatory cytokines, which directly increase the susceptibility to organic angle closure and trabecular dysfunction. TRD was 
Table 2 Logistic regression analysis of the risk factors for requirement of filtration surgery in patients with postoperative neovascular glaucoma after proliferative diabetic retinopathy surgery

\begin{tabular}{|c|c|c|}
\hline & OR $(95 \% \mathrm{CI})$ & $P$-value \\
\hline Male, \% & $1.68(0.15-18.60)$ & 0.67 \\
\hline Age, years & $0.94(0.83-1.05)$ & 0.28 \\
\hline $\operatorname{PRP}(+), \%$ & $0.7 \mathrm{I}(0.06-8.75)$ & 0.79 \\
\hline Presence of lens before vitrectomy, \% & $0.54(0.02-15.82)$ & 0.72 \\
\hline Rubeosis, \% & $1.61(0.10-25.06)$ & 0.73 \\
\hline TRD (+), \% & $0.22(0.01-4.16)$ & 0.31 \\
\hline ME (+), \% & $0.49(0.0 I-17.5 I)$ & 0.70 \\
\hline $\mathrm{VH}(+), \%$ & $0.23(0.01-4.75)$ & 0.34 \\
\hline Use of gas tamponade, $\%$ & $25.5(0.98-668.84)$ & 0.05 \\
\hline Preoperative logMAR & $0.62(0.09-4.10)$ & 0.62 \\
\hline Preoperative IOP, $\mathrm{mmHg}$ & $1.05(0.72-1.52)$ & 0.80 \\
\hline LogMAR after vitrectomy & $0.95(0.24-3.72)$ & 0.94 \\
\hline IOP after vitrectomy, $\mathrm{mmHg}$ & $1.11(0.96-1.27)$ & 0.15 \\
\hline $\mathrm{HbA}_{\mathrm{Ic}}, \%$ & $0.45(0.16-1.33)$ & 0.15 \\
\hline $\mathrm{FBG}, \mathrm{mg} / \mathrm{dL}$ & $1.03(1.00-1.05)$ & 0.03 \\
\hline eGFR, $\mathrm{mL} / \mathrm{min} / 1.73 \mathrm{~m}^{2}$ & $0.98(0.94-1.07)$ & 0.24 \\
\hline
\end{tabular}

Abbreviations: eGFR, estimated glomerular filtration rate; FBG, fasting blood glucose; $\mathrm{HbA}_{\mathrm{Ic}}$, glycated hemoglobin; IOP, intraocular pressure; ME, macular edema; OR, odds ratio; PRP, panretinal photocoagulation; TRD, tractional retinal detachment; $\mathrm{VH}$, vitreous hemorrhage.

not identified as a predictor of filtration surgery in this study. Therefore, gas tamponade should be avoided to reduce the risk of organic angle closure and trabecular dysfunction in the event of NVG in patients with TRD not involving the macula and in patients with no iatrogenic retinal tear. The effects of factors such as the type of gas used for tamponade and the duration of presence of intraocular gas on control of IOP after the onset of NVG should be investigated in future studies.

Our results suggest that a shorter time to onset of NVG is associated with a higher risk of needing filtration surgery, which suggests that these patients have such rapidly progressive NVG that their IOP becomes uncontrollable within a short period.

The assumption that patients with fasting hyperglycemia are more likely to require filtration surgery is based on the

Table 3 Backward elimination method of logistic regression analysis of the risk factors for the requirement of filtration surgery in patients with postoperative neovascular glaucoma after surgery for proliferative diabetic retinopathy

\begin{tabular}{lll}
\hline & OR $(\mathbf{9 5} \% \mathbf{C I})$ & $P$-value \\
\hline Use of gas tamponade, \% & $8.22(1.75-38.52)$ & 0.008 \\
IOP after vitrectomy, mmHg & $1.09(1.00-1.19)$ & 0.04 \\
HbA $_{\text {Ic }} \%$ & $0.50(0.24-1.01)$ & 0.05 \\
FBG, $\mathrm{mg} / \mathrm{dL}$ & $1.03(1.01-1.05)$ & 0.008 \\
\hline
\end{tabular}

Abbreviations: FBG, fasting blood glucose; $\mathrm{HbA}_{1 \mathrm{c}}$, glycated hemoglobin; IOP, intraocular pressure; OR, odds ratio. result of 1 preoperative blood test, so multiple blood tests may be required for more precise data interpretation. Patients with fasting hyperglycemia are more likely to require filtration surgery because endothelial cells are known to be targets for the injurious effects of hyperglycemia. ${ }^{21}$ Microangiopathy caused by fluctuations in blood glucose levels has also been reported to contribute to the progression of ischemia. ${ }^{22}$ In this study, $\mathrm{HbA}_{1 \mathrm{c}}$ was not a significant variable; however, in view of the abovementioned clinical findings, fluctuations in blood glucose levels might have contributed to this result. Further studies are needed to determine if there is a relationship between $\mathrm{HbA}_{1 \mathrm{c}}$ levels and the requirement for filtration surgery.

This study was retrospective in nature and consequently has several limitations that could hamper the interpretation of its findings. First, lens extraction has been identified as a risk factor for $\mathrm{NVG}^{23-25}$ but was not included in this analysis because most patients underwent lens extraction. This study did not find an association between the lens status and the incidence of NVG. One (3\%) of the 40 eyes in the filtration (+) group was phakic after vitrectomy, and none of the 21 eyes in the filtration (-) group was phakic after vitrectomy. Therefore, the number of phakic eyes examined in our study was probably not large enough to detect significant postoperative differences between phakic and pseudophakic eyes. Lens extraction is associated with an increased risk of inflammation of the anterior chamber. Therefore, it is necessary to investigate the association between gas tamponade and filtration surgery in patients not undergoing lens extraction. Second, iris/angle neovascularization may not have been detected before surgery in all cases. Only slit-lamp microscopy or gonioscopy was used to detect iris/angle neovascularization in this series, and fluorescein angiography of the anterior segment or other modalities, for example, spectral-domain anterior segment optical coherence tomography, enabling more accurate detection should be used in future studies. Third, a substantial number of patients did not undergo fluorescein angiography, including those with vitreous hemorrhage or TRD, resulting in inadequate preoperative evaluation of intraocular ischemia. A larger ischemic area is associated with more severe NVG, so it is important to monitor patients continuously for postoperative retinal ischemia using modalities such as fluorescein angiography and optical coherence tomography angiography. Fourth, because we could not determine the total number of preoperative and intraoperative laser shots, the extent of PRP may have varied among the patients in this study. Fifth, some of the patients who underwent filtration surgery could have experienced an IOP decrease because of treatment options other than 
filtration surgery if their NVG had been detected at a very early stage. The time to the first postoperative examination should be decreased for closer monitoring of IOP changes. Sixth, we did not perform gonioscopy at the time that NVG occurred to assess the peripheral anterior synechia index in all cases. Spectral-domain anterior segment optical coherence tomography or gonioscopy enabling more accurate assessment of the peripheral anterior synechia index should be used in future studies.

\section{Conclusion}

The results of this study suggest that preoperative fasting hyperglycemia, IOP at the onset of NVG, and use of gas tamponade during PDR surgery are potential risk factors for requirement of filtration surgery in patients who develop NVG after PDR surgery.

\section{Acknowledgments}

We would like to thank Chiaki Nishimura for statistical consultation and Editage Author Services for editing this manuscript.

\section{Author contributions}

All authors contributed toward data analysis, drafting and revising the paper and agree to be accountable for all aspects of the work.

\section{Disclosure}

The authors report no conflicts of interest in this work.

\section{References}

1. Sima P, Zoran T. Long-term results of vitreous surgery for proliferative diabetic retinopathy. Doc Ophthalmol. 1994;87:223-232.

2. Kumagai K, Furukawa M, Ogino N, Larson E, Iwaki M, Tachi N. Longterm follow-up of vitrectomy for diffuse nontractional diabetic macular edema. Retina. 2009;29:464-472.

3. Goto A, Inatani M, Inoue T, et al. Frequency and risk factors for neovascular glaucoma after vitrectomy in eyes with proliferative diabetic retinopathy. J Glaucoma. 2013;22:572-576.

4. Huang $\mathrm{CH}$, Hsieh YT, Yang CM. Vitrectomy for complications of proliferative diabetic retinopathy in young adults: clinical features and surgical outcomes. Graefes Arch Clin Exp Ophthalmol. 2017;255: 863-871.

5. Kwon JW, Jee D, La TY. Neovascular glaucoma after vitrectomy in patients with proliferative diabetic retinopathy. Medicine (Baltimore). 2017;96:e6263.

6. Scuderi JJ, Blumenkranz MS, Blankenship G. Regression of diabetic rubeosis iridis following successful surgical reattachment of the retina by vitrectomy. Retina. 1982;2:193-196.
7. Wand M, Madigan JC, Gaudio AR, Sorokanich S. Neovascular glaucoma following pars plana vitrectomy for complications of diabetic retinopathy. Ophthalmic Surg. 1990;21:113-118.

8. Kubota T, Aoki R, Harada Y, Tou N, Tawara A. Intravitreal injection of bevacizumab to treat neovascular glaucoma. Jpn J Ophthalmol. 2008; 52:410-412.

9. Yazdani S, Hendi K, Pakravan M, Mahdavi M, Yaseri M. Intravitreal bevacizumab for neovascular glaucoma: a randomized controlled trial. J Glaucoma. 2009;18:632-637.

10. Saito Y, Higashide T, Takeda H, Murotani E, Ohkubo S, Sugiyama K. Clinical factors related to recurrence of anterior segment neovascularization after treatment including intravitreal bevacizumab. Am J Ophthalmol. 2010;149:964-972.e1.

11. Saito Y, Higashide T, Takeda H, Ohkubo S, Sugiyama K. Beneficial effects of preoperative intravitreal bevacizumab on trabeculectomy outcomes in neovascular glaucoma. Acta Ophthalmol. 2010;88:96-102.

12. Every SG, Molteno AC, Bevin TH, Herbison P. Long-term results of Molteno implant insertion in cases of neovascular glaucoma. Arch Ophthalmol. 2006;124:355-360.

13. Yang CM, Yeh PT, Yang CH. Intravitreal long-acting gas in the prevention of early postoperative vitreous hemorrhage in diabetic vitrectomy. Ophthalmology. 2007;114:710-715.

14. Koutsandrea CN, Apostolopoulos MN, Chatzoulis DZ, Parikakis EA, Theodossiadis GP. Hemostatic effects of SF6 after diabetic vitrectomy for vitreous hemorrhage. Acta Ophthalmol Scand. 2001;79:34-38.

15. Balakrishnan D, Jain B, Nayaka A, Rani PK, Mukundaprasad V, Jalali S. Role of tamponade in vitrectomy for proliferative diabetic retinopathy with vitreous hemorrhage. Semin Ophthalmol. 2017;32:488-491.

16. Fujikawa M, Sawada O, Kakinoki M, Sawada T, Kawamura H, Ohji M. Long-term intraocular pressure changes after vitrectomy for epiretinal membrane and macular hole. Graefes Arch Clin Exp Ophthalmol. 2014;252:389-393.

17. Yamamoto K, Iwase T, Terasaki H. Long-term changes in intraocular pressure after vitrectomy for rhegmatogenous retinal detachment, epi-retinal membrane, or macular hole. PLoS One. 2016;11:e0167303.

18. Tripathi RC, Li J, Tripathi BJ, Chalam KV, Adamis AP. Increased level of vascular endothelial growth factor in aqueous humor of patients with neovascular glaucoma. Ophthalmology. 1998;105:232-237.

19. Kim YG, Hong S, Lee CS, et al. Level of vascular endothelial growth factor in aqueous humor and surgical results of Ahmed glaucoma valve implantation in patients with neovascular glaucoma. J Glaucoma. 2009; 18:443-447.

20. Matsuyama K, Ogata N, Jo N, Shima C, Matsuoka M, Matsumura M. Levels of vascular endothelial growth factor and pigment epitheliumderived factor in eyes before and after intravitreal injection of bevacizumab. Jpn J Ophthalmol. 2009;53:243-248.

21. Geraldes P, King GL. Activation of protein kinase C isoforms and its impact on diabetic complications. Circ Res. 2010;106:1319-1331.

22. Lachin JM, Bebu I, Bergenstal RM, et al. Association of glycemic variability in type 1 diabetes with progression of microvascular outcomes in the Diabetes Control and Complications Trial. Diabetes Care. 2017; 40:777-783.

23. Blankenship GW. The lens influence on diabetic vitrectomy results. Report of a prospective randomized study. Arch Ophthalmol. 1980;98: 2196-2198.

24. Rice TA, Michels RG, Rice EF. Vitrectomy for diabetic traction retinal detachment involving the macula. Am J Ophthalmol. 1983;95:22-33.

25. Aaberg TM. Clinical results in vitrectomy for diabetic traction retinal detachment. Am J Ophthalmol. 1979;88:246-253. 


\section{Publish your work in this journal}

Clinical Ophthalmology is an international, peer-reviewed journal covering all subspecialties within ophthalmology. Key topics include: Optometry; Visual science; Pharmacology and drug therapy in eye diseases; Basic Sciences; Primary and Secondary eye care; Patient Safety and Quality of Care Improvements. This journal is indexed on

Submit your manuscript here: http://www.dovepress.com/clinical-ophthalmology-journal
PubMed Central and CAS, and is the official journal of The Society of Clinical Ophthalmology (SCO). The manuscript management system is completely online and includes a very quick and fair peer-review system, which is all easy to use. Visit http://www.dovepress.com/ testimonials.php to read real quotes from published authors. 